\title{
Der Index der Eliten? Politische und mediale Perspektiven auf die nachhaltige Entwicklung in Deutschland
}

\author{
Moritz Clauß
}

Keywords: Indexing, Nachhaltigkeit, Drei-Säulen-Konzept, Grounded Theory

\section{Abstract}

Schreibt die Presse nur das, was die politischen Eliten sagen? Die IndexingHypothese von W. Lance Bennett legt einen solchen Schluss nahe. Diese Arbeit untersucht am Beispiel der Nachhaltigkeitsdebatte in Deutschland eine abgeschwächte Variante der Hypothese, die davon ausgeht, dass die politischen und medialen Darstellungen größtenteils deckungsgleich sind. Zunächst fasst der Beitrag grundlegende Erkenntnisse zum Verhältnis von Politik und Medien sowie zur Entstehung des Nachhaltigkeitskonzepts und der nachhaltigen Entwicklung in Deutschland zusammen. Unter Zuhilfenahme der Grounded Theory untersucht er danach, wie der Themenkomplex »Nachhaltigkeit « in der 229. Sitzung des 18. Deutschen Bundestages vom 31. März 2017 und in den Online-Auftritten deutscher Qualitätszeitungen dargestellt wird. Der Vergleich zeigt, dass die Medienberichterstattung sich oft stark mit der politischen Darstellung überschneidet, teilweise aber deutlich über sie hinausgeht oder hinter ihr zurückbleibt.

Ich danke Ulrich Roos für den Anstoß zu dieser Forschungsarbeit und den jahrelangen offenen und kritischen Austausch.

Moritz Clauß: Der Index der Eliten? Politische und mediale Perspektiven auf die nachhaltige Entwicklung in Deutschland. In: Nils S. Borchers, Selma Güney, Uwe Krüger und Kerem Schamberger (Hrsg.): Transformation der Medien - Medien der Transformation. Verhandlungen des Netzwerks Kritische Kommunikationswissenschaft. Frankfurt am Main: Westend 2021. DOI: https://doi.org/10.53291/YVMY2211.

Moritz Clauß I Journalist I info@moritzclauss.de 
Glauben Sie, was in der Zeitung steht? Laut einer repräsentativen Umfrage von Infratest Dimap (2020) im Auftrag des WDR würden die meisten Menschen in Deutschland auf diese Frage mit »Ja« antworten. Allerdings denken auch 35 Prozent, dass Staat und Regierung den Medien vorgeben, worüber sie berichten sollen. Doch für den Verdacht, dass der deutsche Staat die Presse aktiv kontrolliert, gibt es keine Belege. Was nicht bedeutet, dass die Berichterstattung frei von äußeren Zwängen ist: W. Lance Bennett $(1990,106)$ etwa geht in seiner Indexing-Hypothese davon aus, dass die klassischen Medien meist die unter den politischen Eliten vorherrschenden Standpunkte wiedergeben ganz ohne direkte Beeinflussung. Ob die Presse tatsächlich vor allem den Eliten-Diskurs »indexiert«, also dessen Meinungsspanne anzeigt, untersuche ich im Folgenden am Beispiel der Nachhaltigkeitsdebatte in Deutschland.

\section{Das Verhältnis von Politik und Medien}

Beginnen wir mit einer Kluft, nämlich der zwischen dem journalistischen Anspruch und der Wirklichkeit. Die meisten Journalist*innen beabsichtigen, »die Realität so abzubilden, wie sie ist « (Weischenberg et al. 2006, 102). Pressegesetze und Berufskodizes schreiben eine objektive und wahrheitsgemäße Berichterstattung vor (Neuberger und Kapern 2013, 147-151). Dabei sind Nachrichten immer narrativ und hängen unausweichlich mit sozialen Werten zusammen (Parisi 1997, 673). Die Wissenschaftlerin Cornelia Mothes (2014, 270-271) kommt in einer Studie zu dem Ergebnis, dass Journalist*innen tendenziell Tatsachen den Faktenstatus absprechen, die ihrer eigenen Meinung zuwiderlaufen. Andere Forscher*innen beobachten, dass die Medienberichterstattung regelmäßig Informationen verkürzt darstellt. Sie verweisen zum Beispiel auf eine einseitige Auswahl von Meinungen und auf die bevorzugte Darstellung von Sensationen und negativen Ereignissen (Pfetsch und Marcinkowski 2009, 12). 


\subsection{Einflüsse der politischen Eliten auf die Medien}

Bereits in den 1980er Jahren stellten Edward Herman und Noam Chomsky (1988) ihr Propagandamodell vor. Sie erläutern am Beispiel der USA, welchen äußeren Einflüssen Journalist*innen und Medien ausgesetzt sind. Dabei geht es nicht um eine kontrollierte Meinungsmanipulation, sondern um dezentrale, häufig unbewusste Vorgänge (Krüger 2019, 56). Die beiden Forscher beobachten fünf Filter in der Berichterstattung der US-Medien, darunter die Profitorientierung der Verleger*innen, Werbung als Haupteinkommensquelle und die Abhängigkeit von Informationen, die Regierungen, Unternehmen und Expert*innen zur Verfügung stellen (Herman und Chomsky 1988, 2). Allerdings kritisieren Lang und Lang (2004, 94-97) unter anderem, dass die Beispiele, mit denen Herman und Chomsky ihre Annahmen überprüfen, fast alle aus der US-Außenpolitik stammen. Die Quellenlage ist in diesen Fällen für Journalist*innen häufig schlecht. Aus Sicht seiner Kritiker*innen bleibt deshalb fragwürdig, ob das Modell verallgemeinert werden kann.

Ähnliche Vorhersagen wie das Propagandamodell macht die Indexing-Hypothese von W. Lance Bennett. Sie geht anhand des Beispiels der USA davon aus, dass Medien in der politischen und gesellschaftlichen Debatte vor allem die in der Regierung und in den Parlamenten vorherrschenden Standpunkte wiedergeben (Bennett 1990, 106). Das Meinungsspektrum, das die Medien kommunizieren, ist aus dieser Perspektive ein »Index für die Meinung der politischen Eliten« (Brüggemann und Weßler 2009, 639). Laut Krüger $(2019,54)$ kann eine solche (unbewusst verfolgte) Indexing-Norm unproblematisch sein, solange die politischen Eliten alle relevanten Informationen und Argumente diskutieren - wobei sich natürlich die Frage stellt, woran dies gemessen werden soll.

Laut Bennett (2009) gibt es drei Gründe für Indexing:

• Journalist*innen entwickeln durch tägliche Routinen ein gemeinsames Verständnis davon, wie Quellen und Sichtweisen zu bewerten sind (Bennett 2009, 89). Sie filtern Perspektiven heraus, die als nicht relevant gelten.

- Politiker*innen und ihre Mitarbeiter*innen verstärken diesen Filter, weil sie Informationen an Journalist*innen weitergeben können, die in ihrem Sinne berichten (Bennett 2009, 90). Es besteht ein »Konformitätsdruck«, der auf eine »Anpassung [der Journalist*innen] an 
den Diskursrahmen der politischen Machthaber zielt« (Krüger 2016, 26).

Es entsteht ein Konsens der Eliten, der die Grenzen des öffentlich Denkbaren festlegt. In diesem abgesteckten Rahmen greifen Journalist*innen die Ansichten von Expert*innen und Politiker*innen auf, verbreiten sie und interviewen wiederum letztere dazu. Rezipiert werden diese Beiträge oft von anderen Meinungsführer*innen. Das heißt: Die bestehenden Machtwahrnehmungen und deren Darstellung zementieren sich gegenseitig (Bennett 2009, 90).

Bennett versteht Indexing nicht als allgemeingültiges Gesetz, sondern als Norm, die Tendenzen im Verhalten von Journalist*innen beschreibt (Bennett 1990, 106). Bennett und Livingston $(2003,360)$ distanzieren sich deshalb teilweise von der ursprünglichen Hypothese. Sie bezeichnen Journalist*innen als »semi-independent players« (ebd.), die je nach Fall abhängig oder unabhängig von der Politik handeln. Michael Brüggemann und Hartmut Weßler (2009, 640-642) wiederum halten die von Bennett beobachteten Abhängigkeiten in der Kriegsberichterstattung für gut begründbar, lehnen es jedoch ab, die Medien pauschal als »passive Instrumente« (ebd.) abzustempeln. Bei der Indexing-Hypothese bestehen also ebenfalls Zweifel, ob sie in der Innenpolitik und in friedlichen Szenarien gültig ist.

Im Falle Deutschlands gibt es einige Studien, die analysieren, ob die Medien bei ihrer Berichterstattung von den politischen Eliten abhängig sind. Jürgen Gerhards et al. (1998, 90-95) etwa untersuchen die Berichterstattung der Süddeutschen Zeitung (SZ) und der Frankfurter Allgemeinen Zeitung (FAZ) zum Thema Abtreibung. Sie stellen fest, dass sich die mediale Darstellung stark an der politischen Agenda orientiert. Silke Adam (2008) zeigt mit ihrer Analyse deutscher und französischer Medien von 2000 bis 2002, dass es in Deutschland im Hinblick auf die EU-Erweiterung nationalen politischen Sprecher*innen gelungen ist, »eine innenpolitische Debatte zu vermeiden « (Adam 2008, 137). Sie fügt aber hinzu, dass die mediale Darstellung teilweise über die Darstellung der politischen Eliten hinausgeht (ebd., 137-143).

Torsten Maurer et al. (2008) kommen in ihrer Medienanalyse zu den Kriegen im Kosovo, in Afghanistan und im Irak zu dem Ergebnis, dass Indexing in der deutschen Kriegsberichterstattung eine Leitlinie darstellt. Die Forscher merken aber an, dass ihre Befunde auch davon unabhängig erklärt werden können: Denn die Berichterstattung zu den Kriegen spiegelt nicht nur die Mehrheitsmeinung in den politischen 
Eliten, sondern auch die Mehrheitsmeinung in der deutschen Bevölkerung wider (ebd., 161-165).

Auch Uwe Krüger (2019, 253-258) gelangt in seiner Inhaltsanalyse der Medienberichterstattung über die Münchner Sicherheitskonferenz zu differenzierten Ergebnissen. Demnach orientieren sich die Zeitungen SZ, Welt und FAZ von 2007 bis 2010 deutlich an den politischen und wirtschaftlichen Eliten, bei der tageszeitung (taz) und der Frankfurter Rundschau (FR) ist das hingegen nicht der Fall. In Krügers Analyse der Beiträge von vier einflussreichen Journalisten, die zum Untersuchungszeitpunkt über potenzielle Kontakte in die außen- und sicherheitspolitischen Strukturen der Bundesregierung, der USA oder der NATO eingebunden waren, zeigt sich zudem eine Korrelation zwischen der Berichterstattung dieser Autoren und den Argumentationen US- und NATO-naher Netzwerke. Allerdings stellt auch Krüger fest, dass sich aus der inhaltlichen Nähe politischer und journalistischer Darstellungen kein Kausalzusammenhang ableiten lässt. Möglich wäre etwa, dass die geistige Nähe zwischen Journalist*innen und politischen und wirtschaftlichen Eliten keine Folge, sondern eine Ursache der Berührungspunkte zwischen diesen Gruppen ist.

\subsection{Einflüsse der Medien auf die politischen Eliten}

Wenn wir aber nicht von einfachen Kausalketten ausgehen können, dann bestimmt das Verhältnis zwischen Politiker*innen und Journalist*innen womöglich keine einseitige Abhängigkeit. Wissenschaftler*innen gehen davon aus, dass es sich bei letzteren trotz der aufgezeigten Kritik um Akteur*innen handelt, die aktiv Informationen für die Berichterstattung auswählen, Ereignisse kommentieren und interpretieren (Brüggemann und Weßler 2009, 635; Kabalak et al. 2008, 56; Krüger 2016, 27; Voltmer 2008, 93-94).

Bereits vor einem halben Jahrhundert wurde zudem vermutet, dass die Orientierung der Medien an bestimmten Interessen der Leser*innen langfristig Konsequenzen für Politiker*innen und Parteien haben kann (Gerber 1968, 100). So lässt sich aus dem Akteur-Status der Medien ableiten, dass das Mediensystem den Politiker*innen »seine eigenen Regeln der Aufmerksamkeitserzeugung aufzwingt« (Steiner und Jarren 2009, 251). Wenn diese in der Öffentlichkeit gehört werden wollen, müssen sie die Medien mit Inhalten versorgen, die interessant genug sind, um sie zu veröffentlichen. Politiker*innen handeln 
deshalb unter besonderer Berücksichtigung der Medien. Sie achten etwa darauf, ihre Themen und Vorschläge mediengerecht aufzubereiten und haben dafür eigene PR-Abteilungen. Dazu kommt noch, dass die »Nicht-Informiertheit« bei Politiker*innen eine exkludierende Wirkung entfaltet. Schließlich nutzen sie die mediale Darstellung als »Rückkopplungsinstrument« (Kabalak et al. 2008, 55-59), um herauszufinden, wie bestimmte Themen und Vorschläge bei den Wähler*innen ankommen.

\section{Nachhaltigkeit und die nachhaltige Entwicklung}

Eines der vielen Themengebiete, über das Medien in Deutschland seit Jahren ausführlich berichten, ist Nachhaltigkeit. Egal ob Plastiktüten, Elektroautos oder Corona-Pandemie: Aktuell scheint es kaum noch ein Thema zu geben, das nicht mit der nachhaltigen Entwicklung zusammenhängt. Nur: Was ist das überhaupt, Nachhaltigkeit?

\subsection{Historischer Hintergrund des Nachhaltigkeitsbegriffs}

Viele Forscher*innen sehen den Ursprung des Nachhaltigkeitsbegriffs in der Forstwirtschaft (Soentgen 2016, 117; Giulio 2004, 17; Zürcher 1965, 99). Seine vermutlich erste Verwendung geht auf Hans Carl von Carlowitz zurück (Zürcher 1965, 99), der im Jahr 1713 eine »continuierliche, beständige und nachhaltende [Wald-]Nutzung [sic] « fordert (Carlowitz 1713, 105; zitiert nach Zürcher 1965, 99). Damit meint er, dass dort mindestens so viel Holz nachwachsen soll, wie entfernt wird (Carlowitz 2000, 106-111; zitiert nach Soentgen 2016, 118). Deutsche Historiker*innen und Forstwissenschaftler*innen sind der Auffassung, dass von Carlowitz das Nachhaltigkeitskonzept erfunden hat (Soentgen 2016, 118).

Die Idee, die hinter der nachhaltenden Nutzung steckt, lässt sich allerdings auf das bereits 3000 vor Christus bei den Römer*innen geltende Nießbrauchkonzept zurückführen: usus fructus. Bürger*innen Roms durften bestimmte Dinge - etwa Wälder oder Äcker - nutzen, obwohl sie ihnen nicht gehörten, solange die Substanz der Dinge erhalten blieb (ebd., 118-120). Das Problem dabei: Worin die Substanz, also das Wesentliche einer Sache, besteht, ist Ansichtssache (Rickert 1929, 38). So kann eine Forstwirtin eine ganz andere Vorstellung von 
der Substanz eines Waldes haben als ein Waldökologe (Soentgen 2016, 121-123). Bei vielen Nachhaltigkeitsdefinitionen treffen deshalb unterschiedliche Perspektiven aufeinander - auch bei der wohl wichtigsten, nämlich derjenigen der Vereinten Nationen (UN).

\subsection{Der Nachhaltigkeitsbegriff der Vereinten Nationen}

Die UN sehen einen Zusammenhang zwischen globalen Umweltproblemen und globaler Ungerechtigkeit (Brand und Jochum 2000, 174). Ihre Antwort auf diese Krisen ist das Konzept der nachhaltigen Entwicklung, dem sie sich erstmals im Brundtland-Bericht (1987) und in der Agenda 21 (1992) widmeten. Im Brundtland-Bericht heißt es dazu:

Sustainable development is development that meets the needs of the present without compromising the ability of future generations to meet their own needs. It contains within it two key concepts: the concept of »needs«, in particular the essential needs of the world's poor, to which overriding priority should be given; and the idea of limitations imposed by the state of technology and social organization on the environment's ability to meet present and future needs. (WCED 1987, 41)

Die schützenswerte Substanz bleibt hier weitestgehend unbestimmt. Auffällig ist außerdem, dass der Umwelt kein Eigenwert zugeschrieben wird. Im Vordergrund steht die Funktion der Natur, die menschlichen Bedürfnisse zu befriedigen.

In den Dokumenten der UN-Konferenz 1992 in Rio de Janeiro werden die Begriffe »Nachhaltigkeit« und »nachhaltige Entwicklung « zwar nicht definiert, die UN beziehen sich dort aber auf den Brundtland-Bericht (Giulio 2004, 145). Hiervon ausgehend fasst Antonietta Di Giulio (ebd.) Kernaspekte des UN-Nachhaltigkeitsbegriffs zusammen: Die nachhaltige Entwicklung der Menschheit findet aus UN-Sicht regional, national und global statt. Ihr Ziel ist die Bedürfnisbefriedigung aller Menschen auch zukünftiger Generationen - weltweit. Jedem und jeder soll ein »gutes Leben« ermöglicht werden. Zu diesem Zweck müssen alle Menschen und Staaten partnerschaftlich zusammenarbeiten und die Interessen der anderen respektieren. Am Schluss soll ein weltweiter Konsens entstehen. Nachhaltigkeit wiederum meint im UN-Verständnis den Zustand, der mit der nachhaltigen Entwicklung angestrebt wird. Es handelt sich also um eine in der Zukunft liegende »bessere Welt«, in der keine Widersprüche zwischen dem Sozialen, der Wirtschaft und der Umwelt bestehen (ebd.). 
Ein relevantes aktuelleres Dokument für das UN-Nachhaltigkeitsverständnis ist die »Agenda 2030 für nachhaltige Entwicklung«. Dort benennen die Vereinten Nationen 17 Sustainable Development Goals (SDGs), etwa das weltweite Ende von Armut und Hunger, nachhaltiges Wirtschaftswachstum sowie den Schutz und die nachhaltige Nutzung von Ökosystemen (UN-Generalversammlung 2015, 14). Ein Blick auf die SDGs zeigt: Nachhaltigkeit ist schwer zu erreichen. Die große Zahl der Ziele lässt außerdem offen, welche Bereiche Priorität haben (Samanta 2016). Diese Unklarheiten sind keine Ausnahme, sondern in der Nachhaltigkeitsdebatte die Regel. Denn unterschiedliche Akteur*innen verwenden den Begriff nicht nur oft, sondern oft auch unterschiedlich (Brand und Jochum 2000, 175; Giulio 2004, 11; Brand und Görg 2002, 16). Di Giulio (ebd.) beobachtet eine womöglich inflationäre Verwendung: Sie sieht die Gefahr, dass Nachhaltigkeit zu einem »leeren Modewort zu werden droht«. Immerhin: In der Debatte besteht in zwei Punkten weitestgehend Einigkeit. Erstens gilt die intra- und intergenerationale Gerechtigkeit als ein Kernelement von Nachhaltigkeit. Zweitens wird allgemein anerkannt, dass diese Gerechtigkeit gerade verletzt wird (Bilharz 2008, 328). Keine Einigkeit gibt es hingegen im Hinblick auf die Frage, wer genau die Probleme lösen soll und auf welchem Weg (Bilharz 2005, 5).

\subsection{Die nachhaltige Entwicklung in Deutschland}

In Deutschland verbreitete sich die Rede von der nachhaltigen Entwicklung mit der Agenda 21. Nachhaltigkeit galt lange verstärkt als Mittel zum Zweck, von dem sich Politiker*innen eine verbesserte Wettbewerbsfähigkeit der Wirtschaft erhofften - Stichwort: "nachhaltige[s] Wachstum « (Seefried 2015, 392-395). 2001 berief die rot-grüne Bundesregierung den Rat für Nachhaltige Entwicklung (RNE) ein, der bei der Weiterentwicklung der deutschen Nachhaltigkeitsstrategie helfen und die Regierung beraten soll (RNE 2018). Der Rat definiert die nachhaltige Entwicklung auf seiner Website; Kern sind dort ebenfalls die Generationengerechtigkeit und die gleichberechtigte Trias aus Umwelt, Sozialem und Wirtschaft (RNE 2020). Auf dieses Drei-Säulen-Konzept hatte sich 1993 auch eine Enquête-Kommission des Deutschen Bundestages geeinigt (Brand und Jochum 2000, 75) - die Nähe zur UN-Definition ist offensichtlich.

Unter Bundeskanzlerin Angela Merkel hat das Nachhaltigkeitskonzept in der Bundespolitik deutlich an Gewicht gewonnen (Welfens 
2011, 21-22). Die Bundesregierung hat eine 256 Seiten starke Nachhaltigkeitsstrategie ausgearbeitet, in der sie Nachhaltigkeit als lokales, nationales und globales »Leitprinzip« bezeichnet (Bundesregierung 2017, 17) und sich der Nachhaltigkeitsdefinition des Brundtland-Berichts anschließt (ebd., 24). Im Rahmen einer nachhaltigen Entwicklung fördert sie seit längerem die Elektromobilität (Bundesregierung 2020) und den Ausbau erneuerbarer Energien (Mautz et al. 2008, 42). Im Hinblick auf die Energiewende ist häufig sogar von einer Vorreiterrolle Deutschlands die Rede (Mautz et al. 2008, 12; Welfens 2011, 23).

Das wissenschaftliche Fundament der deutschen Nachhaltigkeitspolitik ist die Theorie der Ökologischen Modernisierung: Neue Technologien sollen den Schutz der Umwelt sicherstellen und zu wirtschaftlichem Erfolg beitragen (Seefried 2015, 395). Da der Anteil deutscher Umwelt- und Klimaschutztechnologien auf dem Weltmarkt hoch ist, gilt die Energiewende als Chance für Deutschland und die deutsche Wirtschaft (Schafhausen 2013, 15). Allerdings betonen manche Ökonom*innen, dass die Anhänger*innen des grünen Wachstums mit unrealistischen Umwelttechnologie-Sprüngen rechnen (Paqué 2012, 1718). Andere halten fest, dass weniger umweltschädliche Technologien nicht automatisch zum Umweltschutz beitragen. Das Problem ist der Rebound-Effekt: Effizienzsteigerungen beim Energie- oder Ressourcenverbrauch werden häufig durch eine steigende Nachfrage aufgefressen (Santarius 2012, 5-6).

\section{Methodik und Forschungsfrage}

In der folgenden Analyse zeige ich, welche Gemeinsamkeiten und Differenzen sich in den Darstellungen des Themenkomplexes Nachhaltigkeit durch die politischen Eliten und durch die Online-Angebote von Qualitätszeitungen beobachten lassen. Im Gegensatz zur ursprünglichen Indexing-Hypothese beschäftige ich mich nicht mit kausalen Mechanismen, weil die hier vorgestellten Studien belegen, dass eine einseitige Auslegung des Verhältnisses von Politik und Medien in Deutschland nicht haltbar ist. Meine schwächere Variante der Hypothese lautet, dass die politischen und medialen Darstellungen größtenteils deckungsgleich sind. Diese Aussage bleibt relevant, weil ich wie Dahrendorf $(1968,17)$ und Krüger $(2019,53)$ davon ausgehe, dass die Medienberichterstattung in einer Demokratie grundsätzlich über die Debatte im politischen Zentrum hinausgehen sollte. Aufgabe des Jour- 
nalismus ist es auch, Optionen aufzuzeigen, die von den politischen Entscheider*innen ignoriert oder übersehen werden (ebd.). Da die Nachhaltigkeitsdebatte alle Menschen betrifft, ist eine breite, ausgewogene und offene Berichterstattung in diesem Fall besonders wichtig.

Als Methodologie greife ich auf eine Variante der Grounded Theory zurück, die auf Vorarbeiten von Jörg Strübing (2008) sowie von Ulrich Franke und Ulrich Roos (2010) basiert. Die Grounded Theory verfolgt einen rekonstruktiven Ansatz und setzt eine möglichst große Offenheit der Forscher*innen gegenüber dem Untersuchungsgegenstand voraus (Franke und Roos 2010, 285). Qualitative Analyseschritte finden abwechselnd statt und beeinflussen sich gegenseitig. Forscher*innen kodieren das Datenmaterial, entwickeln Vermutungen, interpretieren und suchen nach relevanten Zusammenhängen. Spontane Beobachtungen und Thesen sollen in Form von Memos erhalten bleiben. Im Lauf der Analyse zeigt sich dann, ob eine These zu halten ist oder verworfen werden muss (Strübing 2008, 14).

Zu den politischen Eliten zählen in der Regel »alle unmittelbar an der Staatsleitung beteiligten Personen und Gruppen, unabhängig davon, ob sie sich bei einzelnen Entscheidungen durchsetzen oder nicht « (Herzog und Weßels 2005, 732). Ich fasse den Begriff etwas weiter und beziehe ihn auf alle Politiker*innen, die im Analysezeitraum ein Mandat im Deutschen Bundestag innehatten. Das schließt die Opposition mit ein. Im Falle der politischen Eliten untersuche ich die Nachhaltigkeitsdebatte in der 229. Sitzung des 18. Deutschen Bundestages, die am 31. März 2017 stattfand (Deutscher Bundestag 2017). Darin beziehen alle zum damaligen Zeitpunkt im Parlament vertretenen Parteien Stellung zur deutschen Nachhaltigkeitsstrategie. Ich gehe davon aus, dass die jeweiligen Sprecher*innen im Rahmen der Debatte sämtliche Punkte ansprechen, die aus Sicht ihrer politischen Zugehörigkeit wichtig sind.

$\mathrm{Zu}$ den wichtigsten deutschen Qualitätszeitungen werden meist die Frankfurter Allgemeine Zeitung (FAZ), Die Welt, die Süddeutsche Zeitung (SZ), die Frankfurter Rundschau (FR) und die tageszeitung (taz) gezählt (Lüter 2008, 117; Jandura und Kösters 2017, 30-31; Krüger 2019, 232). Ich schließe mich dieser Auswahl an. ${ }^{1}$ Die Zeitungen lassen sich in

1 Offenlegung: Im Jahr 2016 habe ich zwei Monate in der Onlineredaktion der taz gearbeitet. Ich sehe hierin keinen Interessenkonflikt, weil die Arbeitsbeziehung zum Analysezeitpunkt mehr als ein Jahr zurücklag und ich in der Analyse keine Texte von mir persönlich bekannten Autor*innen untersucht habe. Die Auswahlkriterien für die taz-Texte unterschieden sich nicht von denen für die anderen Zeitungen. 
einem Links-Rechts-Schema anordnen: Artikel in der taz und der FR vertreten vergleichsweise häufig linke, Artikel in der $F A Z$ und der Welt oft konservative bis rechte Positionen. Die $S Z$ tendiert eher zu linken Positionen, positioniert sich aber insgesamt ausgewogener als der Rest des Feldes (Lüter 2008). In der Analyse werden Texte der Online-Angebote dieser Zeitungen zum Thema Nachhaltigkeit analysiert, die maximal sechs Monate vor oder nach der Bundestagsdebatte erschienen sind. Hinzu kommen zwei weitere Auswahlkriterien: der Inhalt und seine Gewichtung. Über die Suchfunktionen der Zeitungswebsites habe ich nach Artikeln zum Stichwort »Nachhaltigkeitsstrategie « gesucht; bei einer geringen Zahl von Treffern folgten außerdem Suchen nach »Nachhaltigkeit« und »nachhaltig«. Ins Datenmaterial aufgenommen wurden die Beiträge, die sich am ausführlichsten dem Thema Nachhaltigkeit widmeten. Insgesamt habe ich 20 Zeitungsartikel (Tab. 1) analysiert, also vier Artikel pro Zeitung. Diese Zahl sollte groß genug sein, um einen aussagekräftigen Überblick über die mediale Darstellung zu erhalten.

Jeweils vier Artikel sind meiner Ansicht nach aber zu wenig, um die Berichterstattung der einzelnen Zeitungen wertend zu vergleichen. Die Kategorisierung greift auf die verschiedenen Positionen zurück, die in den Artikeln wiedergegeben werden. Da mitunter in einem Zeitungsbeitrag mehrere gegenläufige Aussagen enthalten sind, kann ein Artikel auch verschiedene, sich widersprechende Analysekategorien stützen. Bei der Einordnung wird außerdem nicht zwischen Textgattungen wie Bericht, Meldung oder Kommentar unterschieden. Wichtig ist für die vorliegende Analyse nicht, ob der oder die Journalist*in eine bestimmte Meinung oder Position vertritt, sondern welche Meinungen und Positionen in den Artikeln dargestellt werden. Wenn ich im Folgenden von »medialen Darstellungen« schreibe, folgt daraus also nicht automatisch, dass alle untersuchten Artikel oder Zeitungen die entsprechende Darstellung stützen.

Tab. 1: Liste der analysierten Medienartikel.

\begin{tabular}{|l|l|l|l|}
\hline Titel & Autor*in & Medium & Datum \\
\hline $\begin{array}{l}\text { Nachhaltige Investments werden für Anleger } \\
\text { immer wichtiger }\end{array}$ & $\begin{array}{l}\text { Christoph } \\
\text { Scherbaum }\end{array}$ & FAZ.net & 01.06 .2017 \\
\hline IHK entdeckt die Nachhaltigkeit & FAZ.net & FAZ.net & 15.02 .2017 \\
\hline
\end{tabular}




\begin{tabular}{|c|c|c|c|}
\hline Titel & Autor*in & Medium & Datum \\
\hline Alles nachhaltig oder was? & Thomas Klemm & FAZ.net & 11.08 .2017 \\
\hline Ist Nachhaltigkeit nur ein Schlagwort? & Elena Witzeck & FAZ.net & 17.09.2017 \\
\hline $\begin{array}{l}\text { Mehr leben. Nachhaltiges Konsumieren, geht } \\
\text { das überhaupt? }\end{array}$ & Christine Ax & FR.de & 12.05 .2017 \\
\hline $\begin{array}{l}\text { Nachhaltigkeit ist auch beim Autobau ein } \\
\text { wichtiges Thema }\end{array}$ & Fabian Hoberg & FR.de & 30.12 .2016 \\
\hline Zwischen heiter und wolkig & Daniela Vates & FR.de & 11.01.2017 \\
\hline Wem gehört der letzte Tropfen? & Joachim Wille & FR.de & 13.06.2017 \\
\hline Prekäre Erkenntnis & $\begin{array}{l}\text { Michael } \\
\text { Bauchmüller }\end{array}$ & SZ.de & 24.01.2017 \\
\hline Verzichten können andere & $\begin{array}{l}\text { Michael } \\
\text { Bauchmüller und } \\
\text { Kristiana Ludwig }\end{array}$ & SZ.de & 16.03 .2017 \\
\hline Die Leiden des aufgeklärten Konsumenten & $\begin{array}{l}\text { Michael } \\
\text { Kläsgen }\end{array}$ & SZ.de & 01.01 .2017 \\
\hline Mehr als grünes Geschwätz & Jan Willmroth & SZ.de & 25.06 .2017 \\
\hline Party ohne Ende & Edith Kresta & taz.de & 19.03.2017 \\
\hline Erfolg durch schwache Kriterien & $\begin{array}{l}\text { Malte } \\
\text { Kreutzfeldt }\end{array}$ & taz.de & 11.01.2017 \\
\hline Mehlwurm statt Mehrwert & Bernhard Pötter & taz.de & 30.05 .2017 \\
\hline Interdisziplinärer Thinktank & $\begin{array}{l}\text { Manfred } \\
\text { Ronzheimer }\end{array}$ & taz.de & 12.05 .2017 \\
\hline »Nachhaltig « ist unser »halal« & Matthias Heine & Welt.de & 05.06 .2017 \\
\hline Was bringen Ökolabels im Tourismus? & Steven Hille & Welt.de & 09.06 .2017 \\
\hline $\begin{array}{l}\text { „Das Immer-mehr führt auf Dauer zum } \\
\text { Kollaps« }\end{array}$ & Frank Lorentz & Welt.de & 07.06.2017 \\
\hline $\begin{array}{l}\text { So geht Deutschland Klima-China auf den } \\
\text { Leim }\end{array}$ & Daniel Wetzel & Welt.de & 02.06 .2017 \\
\hline
\end{tabular}




\section{Ergebnisse der Analyse}

In der Analyse habe ich sechs Kategorien gebildet, die sich jeweils aus mehreren Subkategorien zusammensetzen. In den folgenden Abschnitten gehe ich einzeln auf diese ein.

\subsection{Dominanz des Nachhaltigkeitsverständnisses der UN}

Die Analyse zeigt, dass die politischen Eliten Nachhaltigkeit fast ausschließlich im Sinne des UN-Verständnisses darstellen. Die drei Nachhaltigkeitssäulen Wirtschaft, Soziales und Umwelt werden von allen politischen Akteur*innen als Bestandteile einer nachhaltigen Entwicklung angesehen. Die Parteien heben teilweise zwar unterschiedliche Aspekte hervor - etwa betonen Linke, Grüne und SPD im Gegensatz zur Union, dass der Faktor Gerechtigkeit besonders wichtig ist -, verlassen dabei aber nicht den durch die UN gesteckten Rahmen: Kein*e Politiker*in geht eindeutig über die 17 SDGs hinaus oder wendet sich gegen sie. Die analysierten Medien verbreiten größtenteils ebenfalls das Nachhaltigkeitsverständnis der UN - häufig jedoch, ohne es direkt zu benennen.

In der Bundestagsdebatte betonen mehrere Redner*innen, dass es eine besonders positive und konstruktive politische Zusammenarbeit in der nachhaltigen Entwicklung gibt. Sowohl die Bundesregierung als auch die im Parlament vertretenen Parteien stufen die nachhaltige Entwicklung außerdem im Sinne des UN-Begriffs als alternativlos ein:

Die Verankerung des Prinzips der nachhaltigen Entwicklung als Leitprinzip in allen Politikbereichen ist gerade in weltpolitisch schwierigen Zeiten kein Luxus, sondern pure Notwendigkeit. (Altmaier; zitiert nach Deutscher Bundestag 2017, 4)

Dieser Alternativlosigkeit-Frame wird auch in vielen Medienberichten dargestellt. Im Gegensatz zu den Politiker*innen geben die Medien aber auch der entgegengesetzten Annahme Raum: Sie stellen Nachhaltigkeit teilweise als eine (politische) Option unter anderen, stellenweise sogar als schädlich dar und verweisen auf eine mangelhafte wissenschaftliche Überprüfbarkeit der vorgebrachten Thesen. So schreibt die Welt zur Nachhaltigkeitsdefinition des Brundtland-Berichts: »In ihr deutet sich schon die quasireligiöse Verschwurbelung des Begriffs Nachhaltigkeit an [...]« (Heine 2017). Den Frame der besonders positiven politischen Kooperation greifen die Medien außerdem nicht auf. 
Tab. 2: Zusammenfassung der Analysekategorie »Dominanz des Nachhaltigkeitsverständnisses der UN «.

\begin{tabular}{|l|l|l|}
$\begin{array}{l}\text { Gemeinsame } \\
\text { Darstellungen }\end{array}$ & $\begin{array}{l}\text { Breiter Nachhaltigkeitsbegriff auf Grundlage des UN-Nachhaltigkeitsver- } \\
\text { ständnisses; ungeklärtes normatives Verhältnis der drei Nachhaltigkeits- } \\
\text { säulen; Darstellung der nachhaltigen }\end{array}$ \\
\hline $\begin{array}{l}\text { Entwicklung als alternativlos. } \\
\text { Darstellungen }\end{array}$ & $\begin{array}{l}\text { Medien: Darstellung der nachhal- } \\
\text { tigen Entwicklung als nicht alter- } \\
\text { nativlos; Thematisierung weiterer } \\
\text { Nachhaltigkeitsvorstellungen. }\end{array}$ & $\begin{array}{l}\text { Politik: Betonung einer besonders } \\
\text { guten politischen Zusammenarbeit; } \\
\text { Betrachtung der nachhaltigen } \\
\text { Entwicklung als Phänomen, das } \\
\text { national und global vorangetrieben } \\
\text { werden muss. }\end{array}$ \\
\hline Einordnung & Kein Indexing. & \\
\hline
\end{tabular}

Mehrere Politiker*innen betonen, dass die nachhaltige Entwicklung national und global vorangetrieben werden muss. Es entsteht der Eindruck, dass sie versuchen, sich, ihre Partei oder ihr Land besonders positiv erscheinen zu lassen, indem sie große Handlungsbereitschaft und Verantwortungsbewusstsein kommunizieren. Die Medien gehen nicht auf diesen möglichen Versuch der Selbstprofilierung ein - sie unterstützen ihn nicht, üben aber auch keine Kritik daran.

Die Auswertung der Bundestagsdebatte zeigt zudem, dass das normative Verhältnis der drei Nachhaltigkeitssäulen bisher ungeklärt ist. Die drei Bereiche der nachhaltigen Entwicklung werden zwar von allen politischen Akteur*innen grundsätzlich anerkannt, es wird aber noch darüber gestritten, wie genau diese auszubalancieren sind - die Bundesregierung und die Vertreter*innen der Union sprechen von »Zielkonflikte[n] « (Deutscher Bundestag 2017, 5, 6, 7, 10). Die Union warnt vor einer Dominanz der ökologischen Säule und vertritt das Primat des Wachstums. Linke und Grüne rücken den Klimaschutz als Ausgangspunkt einer effektiven Armutsbekämpfung in den Vordergrund. Diese Zielkonflikte spielen auch in der Medienberichterstattung eine Rolle. Die analysierten Zeitungen stellen die Konflikte - etwa zwischen Ökonomie und Ökologie - allerdings umfassender dar als die Politiker*innen.

Die Medien thematisieren zudem weitere Nachhaltigkeitsvorstellungen, mit denen sie über die politische Darstellung hinausgehen. So wird der UN-Nachhaltigkeitsbegriff zwar so gut wie gar nicht direkt kritisiert, wohl aber werden alternative Konzepte aufgezeigt; etwa be- 
sonders weiche Definitionen, denen zufolge es bereits nachhaltig ist, keine geächteten Firmen zu unterstützen. Mehrfach hinterfragen die Zeitungsartikel außerdem direkt oder indirekt, ob einheitliche, allgemein anerkannte Nachhaltigkeitskataloge möglich sind:

Das Siegel des Tourism Sustainability Council (TSC) sollte Nachhaltigkeitskriterien für alle touristischen Angebote der Welt enthalten und nachhaltiges Reisen vereinfachen. Das Vorhaben scheiterte. Denn ein Campingplatz in Österreich zum Beispiel hat ganz andere Grundvoraussetzungen als eine Eco-Lodge im Dschungel von Borneo. (Hille 2017)

Reines Indexing lässt sich in dieser Analysekategorie somit nicht beobachten. Auffällig ist allerdings, dass das Nachhaltigkeitsverständnis der UN in der Berichterstattung weit verbreitet ist, aber kaum direkt reflektiert oder kritisiert wird.

\subsection{Nachhaltigkeit als Mittel zur Interessenverfolgung}

Die Bundesregierung und die Union zeigen in der Debatte, dass sie die nachhaltige Entwicklung für ein umweltverträgliches Wirtschaftswachstum nutzen möchten. Der Umweltschutz wird dabei teilweise dem wirtschaftlichen Erfolg untergeordnet. Auch die Medienberichterstattung stellt eine enge Verknüpfung zwischen diesen beiden Zielen dar. Während die politischen Eliten eher die gesamte Volkswirtschaft oder den Staat im Blick haben, konzentrieren sich die analysierten Artikel auf den wirtschaftlichen Nutzen für Unternehmen und Investor*innen. Die $S Z$ zitiert etwa Wolfgang Fink, den Deutschland-Chef von Goldman Sachs, mit den Worten:

Der ganze Komplex ESG [Environmental, Social and Corporate Governance; MC] wird als Instrument des Risikomanagements unterschätzt. [...] Wir können inzwischen herauslesen, welche Faktoren am stärksten mit dem langfristigen Erfolg einer Firma korrelieren. (zitiert nach Willmroth 2017)

Während die Politiker*innen an diesem Punkt der Verknüpfung von nachhaltiger Entwicklung und wirtschaftlichem Erfolg stehen bleiben, gehen die Medien deutlich darüber hinaus. Sie zeigen ausführlich, dass Unternehmen das Stichwort »Nachhaltigkeit« häufig zur Imagepflege nutzen oder gar missbrauchen. Zentral ist der Vorwurf des Greenwashings. 
Tab. 3: Zusammenfassung der Analysekategorie »Nachhaltigkeit als Mittel zur Interessenverfolgung".

\begin{tabular}{|c|c|c|}
\hline Gemeinsame & \multicolumn{2}{|c|}{ Nachhaltigkeit soll zum wirtschaftlichen Erfolg beitragen. } \\
\hline $\begin{array}{l}\text { Exklusive } \\
\text { Darstellungen }\end{array}$ & $\begin{array}{l}\text { Medien: Nachhaltigkeit als Label } \\
\text { zur Imageförderung; übertrieben } \\
\text { positive Selbstdarstellung von Un- } \\
\text { ternehmen und ihren Leistungen. }\end{array}$ & $\begin{array}{l}\text { Politik: Umstrittener Vorreiterstatus } \\
\text { Deutschlands. }\end{array}$ \\
\hline Einordnung & Kein Indexing. & \\
\hline
\end{tabular}

Der im Forschungsstand angesprochene Vorreiterstatus Deutschlands in der nachhaltigen Entwicklung ist in der Parlamentsdebatte umstritten: Die Bundesregierung und beide Regierungsparteien framen Deutschland als international führende Kraft und stellen das deutsche Handeln als besonders positiv, verantwortungsvoll und hilfreich dar, während die Opposition wiederum aufzeigt, dass Politik und Wirtschaft hierzulande teilweise alles andere als nachhaltig handeln. Die analysierten Medien greifen die Debatte um den Vorreiterstatus nicht direkt auf, widersprechen jedoch stellenweise indirekt dem VorreiterFrame, indem sie Kritikpunkte an der Bundespolitik benennen. Auch diese Kategorie widerspricht der Indexing-Hypothese.

\subsection{Gemeinsame Verantwortung für die nachhaltige Entwicklung}

In der politischen Debatte gilt die Annahme, dass eine nachhaltige Entwicklung in der gemeinsamen Verantwortung von Politik, Wirtschaft, Wissenschaft und Gesellschaft liegt. Die Medienberichterstattung kommuniziert dieselbe gesamtgesellschaftliche Verantwortung. In der Bundestagsdebatte legen die Politiker*innen häufig, aber meist nicht konkret dar, welche Aufgaben die (Bundes-)Politik in der nachhaltigen Entwicklung erfüllen sollte; etwa die Mitfinanzierung dieser Entwicklung und ihre Förderung in mehreren Bereichen (beispielsweise Umwelt-, Wirtschafts- und Außenpolitik).

Mehrfach betonen Redner*innen, dass Bürger*innen im Zuge der nachhaltigen Entwicklung gebildet und informiert werden müssen. Die Oppositionsparteien fordern die Regierung zudem dazu auf, mehr 
Druck auf Behörden, aber auch auf Unternehmen und Konzerne im Dienst der nachhaltigen Entwicklung auszuüben. In der Medienberichterstattung werden ebenfalls die Faktoren Bildung, Information sowie gesetzlicher Druck benannt; sie sollen die nachhaltige Entwicklung vorantreiben. Auch die Förderung durch Investitionen ist Thema dieses Diskurses. Ähnlich wie Oppositionsvertreter*innen schreiben mehrere Journalist*innen dem Staat ein bisher geringes Interesse an nachhaltigem Handeln zu. So schreibt die $S Z$ über eine Reform des Vergaberechts, das Vorgaben für öffentliche Aufträge macht:

[E]ine Gesamtstrategie für nachhaltigen Staatskonsum entstand dabei nicht. Den Behörden wurde lediglich erlaubt, ihre Aufträge auch an soziale oder umweltbezogene Standards zu koppeln. Ein freiwilliger Schritt, auf den jede Kommune, die unter Spardruck steht, auch verzichten kann. (Bauchmüller und Ludwig 2017)

Tab. 4: Zusammenfassung der Analysekategorie »Gemeinsame Verantwortung für die nachhaltige Entwicklung«.

\begin{tabular}{|l|l|l|}
$\begin{array}{l}\text { Gemeinsame } \\
\text { Darstellungen }\end{array}$ & $\begin{array}{l}\text { Politik, Wirtschaft, Wissenschaft und Gesellschaft sollen die nachhaltige } \\
\text { Entwicklung gemeinsam vorantreiben; Politik als verbindendes Glied in der } \\
\text { nachhaltigen Entwicklung. }\end{array}$ \\
\hline $\begin{array}{l}\text { Exklusive } \\
\text { Darstellungen }\end{array}$ & Medien: Keine. & Politik: Keine. \\
\hline Einordnung & Indexing.
\end{tabular}

Insgesamt bleibt die Beschreibung konkreter Aufgabenfelder der Politik in beiden Fällen diffus. Klare Forderungen finden sich sowohl in der politischen Debatte als auch in den analysierten Zeitungen kaum. Sowohl die politische als auch die mediale Darstellung rahmen die Politik als verbindendes Glied, das nachhaltiges Handeln aller Akteur*innen fördern oder ermöglichen kann. In diesem Fall verlaufen beide, gemäß der Indexing-Hypothese, größtenteils deckungsgleich.

\subsection{Positive Bewertung der nachhaltigen Entwicklung}

In der politischen Debatte stellen die Bundesregierung und die Regierungsparteien den Verlauf der nachhaltigen Entwicklung in Deutsch- 
land äußerst positiv dar, etwa wenn SPD-Redner Carsten Träger sagt, die Regierung habe »wirklich hervorragende Arbeit geleistet « (zitiert nach Deutscher Bundestag 2017, 8). Kritik an der Regierungspolitik blenden sie zwar nicht aus, rücken sie jedoch in den Hintergrund oder stellen sie als haltlos - oder politisch motiviert - dar:

Wir haben - das ist der wesentliche Ansatz, dem die Politik im Augenblick folgt - auch die Mittel zur Klimaschutzfinanzierung über das BMZ - mittlerweile 2 Milliarden Euro - verdoppelt. [...] Frau Göring-Eckardt, ich glaube nicht, dass Sie, wenn Sie diese Dinge bewusst übersehen, Ihrer Fraktion und Ihrer Partei einen Gefallen tun. (Marschall; zitiert nach Deutscher Bundestag 2017, 13)

Tab. 5: Zusammenfassung der Analysekategorie »Positive Bewertung der nachhaltigen Entwicklung«.

\begin{tabular}{|l|l|l|}
\hline $\begin{array}{l}\text { Gemeinsame } \\
\text { Darstellungen }\end{array}$ & $\begin{array}{l}\text { Verlauf der nachhaltigen Entwicklung ist grundsätzlich positiv; Vereinbar- } \\
\text { keit ökologischer, sozialer und ökonomischer Interessen ist möglich. }\end{array}$ \\
\hline $\begin{array}{l}\text { Exklusive } \\
\text { Darstellungen }\end{array}$ & $\begin{array}{l}\text { Medien: Nachhaltigkeitsgedanke } \\
\text { kann aufgrund seiner weiten Ver- } \\
\text { breitung die nachhaltige Entwick- } \\
\text { lung in Deutschland vorantreiben; } \\
\text { freier Markt trägt seinen Teil zur } \\
\text { nachhaltigen Entwicklung bei. }\end{array}$ \\
\hline Einordnung & Kein Indexing. \\
\hline
\end{tabular}

Die analysierten Medienbeiträge beziehen sich oft direkt auf Aussagen von Politiker*innen und greifen deshalb auch die positive Darstellung der nachhaltigen Entwicklung von Regierung und Regierungsparteien auf. Während einige Parlamentarier*innen betonen, dass die Entwicklung durch Information und Weiterbildung der Bürger*innen vorangetrieben werden kann, sehen die Medien hier bereits erste Erfolge: Mehrfach findet sich in der medialen Darstellung die Perspektive, dass der in Deutschland bereits weit verbreitete Nachhaltigkeitsgedanke die nachhaltige Entwicklung vorantreiben kann. Die Berichterstattung thematisiert eine Macht der Konsument*innen über die Konzerne. Sowohl in der politischen als auch in der medialen Darstellung wird zudem mehrfach die Vereinbarkeit der drei Nachhaltigkeitsbereiche als möglich eingestuft. 
Häufig stellen die analysierten Medien die Position dar, dass der freie Markt seinen Teil zur nachhaltigen Entwicklung beiträgt. Zentral ist hierbei die Annahme, dass die Umsetzung ökologischer und sozialer Interessen den wirtschaftlichen Interessen dienen kann - und umgekehrt:

Tourismus, so die Welttourismusorganisation (UNWTO), sei ein Instrument zur Abschaffung der Armut, zum Schutz der Umwelt, zur Verbesserung der Lebensqualität und zur wirtschaftlichen Stärkung von Frauen und Jugendlichen. (Kresta 2017)

In diesem Fall handelt es sich um eine Sichtweise, die von der Autorin Edith Kresta zwar festgehalten, im Laufe des Artikels aber abgelehnt wird. Ähnliche mediale Darstellungen führen bis zur These, dass nachhaltigere Unternehmen oft auch erfolgreichere Unternehmen sind. Die politischen Eliten thematisieren die positive Bedeutung des freien Marktes für die nachhaltige Entwicklung hingegen kaum. Das gilt auch für die Annahmen, dass der Nachhaltigkeitsgedanke in Deutschland bereits etabliert ist und dass seine Verbreitung sich bereits positiv auf die nachhaltige Entwicklung auswirkt. Trotz der vielen Überschneidungen der politischen und medialen Darstellungen widerspricht diese Kategorie somit der Indexing-Hypothese.

\subsection{Kritische Bewertung der nachhaltigen Entwicklung}

Den positiven Bewertungen des Verlaufs der nachhaltigen Entwicklung stehen die negativen gegenüber. Sowohl in der oppositionellen als auch in der medialen Darstellung findet sich die Ansicht, dass der freie Markt bisher kaum oder gar nicht zu einer nachhaltigen Entwicklung der Wirtschaft beigetragen hat. So werfen etwa die Linken der Regierung vor, in Sachen Nachhaltigkeit fälschlicherweise auf freiwilliges Handeln zu setzen. Die Medienberichterstattung kommuniziert diese Perspektive ebenfalls - und zwar deutlich ausführlicher als die Opposition. In den Zeitungsartikeln findet sich wiederholt der Standpunkt, dass Anleger*innen und Unternehmen kein großes Interesse an nachhaltigen Investitionen haben. Somit berichten die Medien nicht nur ausführlicher über die Möglichkeiten des freien Marktes, sondern geben auch der Kritik an diesem Konzept deutlich mehr Raum als die politischen Eliten. Die Einflussmöglichkeiten der Einzelnen werden hierbei nicht generell bestritten. Laut der Berichterstattung nutzen die meisten Menschen diese Möglichkeiten aber noch nicht oder kaum: 
Es gebe zwar »einen Trend zum Individualtourismus«, berichtete Friederike Grupp, die Nachhaltigkeitsbeauftrage des Touristik-Unternehmens Thomas Cook. Doch die meisten Menschen wollten eben »an den Strand«. Der Badetourismus, ob am Mittelmeer oder zunehmend auch in fernen Ländern, bleibe der Schwerpunkt. (Wille 2017)

Die $S Z$ thematisiert außerdem, dass es für Individuen sehr schwierig sein kann, nachhaltig zu handeln. Das Beispiel ist in diesem Fall der nachhaltige Konsum:

Nie war Einkaufen so kompliziert, es gibt zu viele Fragen, die sich kaum beantworten lassen. Der moderne Konsument rennt im Hamsterrad seiner eigenen Ansprüche. (Kläsgen 2017)

Wer nachhaltig konsumieren will, benötigt dafür viele miteinander zusammenhängende Informationen und muss diese auch auswerten können. Die Medienberichterstattung zeigt an vielen Stellen auf, dass bisher unklar ist, welchen Einfluss Individuen tatsächlich durch ihren Konsum auf die nachhaltige Entwicklung haben. In der Bundestagsdebatte spielt diese Frage wiederum kaum eine Rolle. Mehrere der untersuchten Zeitungsartikel beschäftigen sich außerdem direkt oder indirekt mit der Frage, ob sich Nachhaltigkeit und Konsum nicht grundsätzlich widersprechen.

Die Berichterstattung geht auch darauf ein, dass weniger Konsum nicht automatisch weniger Zufriedenheit oder Lebensqualität bedeuten muss. In der politischen Debatte nimmt das Thema Verzicht eine weitaus kleinere Rolle ein. Seine Notwendigkeit wird zwar stellenweise angedeutet, er selbst aber nur in einem einzigen Fall konkret gefordert: Linken-Politikerin Katja Kipping will weniger Verkehr auf den Straßen (Deutscher Bundestag 2017, 5).

Die analysierten Medien greifen zudem immer wieder die Annahme auf, dass die nachhaltige Entwicklung nur erfolgreich sein kann, wenn Perspektivwechsel stattfinden. Neben dem Verzicht auf Konsumgüter thematisiert die Medienberichterstattung auch eine mögliche Reduzierung der Arbeitszeit oder das Essen von Insekten als Alternative zum Fleischkonsum. In der politischen Debatte finden sich solche Perspektivwechsel meist nur als abstrakte Schlagworte ohne Erklärung - etwa »Paradigmenwechsel« (Strässer; zitiert nach Deutscher Bundestag 2017, 14) oder »Transformationsauftrag « (Träger; zitiert nach Deutscher Bundestag 2017, 8). 
Tab. 6: Zusammenfassung der Analysekategorie »Kritische Bewertung der nachhaltigen Entwicklung«.

\begin{tabular}{|l|l|}
\hline $\begin{array}{l}\text { Gemeinsame } \\
\text { Darstellungen }\end{array}$ & $\begin{array}{l}\text { Freier Markt hat bisher kaum zu einer nachhaltigen Entwicklung der Wirt- } \\
\text { schaft beigetragen; Nachhaltigkeitspolitik der Bundesregierung ist noch } \\
\text { nicht erfolgreich. }\end{array}$ \\
\hline Exklusive & $\begin{array}{l}\text { Medien: Nachhaltiges Handeln der } \\
\text { Darstellungen }\end{array}$ \\
& $\begin{array}{l}\text { Individuen ist hierzulande nicht } \\
\text { etabliert; Verzicht als Alternative } \\
\text { zum nachhaltigen Konsum; } \\
\text { Notwendigkeit von Perspektiv- } \\
\text { wechseln, damit die nachhaltige } \\
\text { Entwicklung erfolgreich sein kann. }\end{array}$ \\
\hline Einordnung & Kein Indexing. \\
\hline
\end{tabular}

Artikel der FR, der $S Z$ und der taz bewerten die Nachhaltigkeitspolitik der Regierung als nicht besonders erfolgreich. Unter anderem wird kritisiert, dass man keine großen Veränderungen anstrebe. Die von der Regierung kommunizierten Erfolge werden in der Mediendarstellung mitunter auf angepasste Nachhaltigkeitskriterien zurückgeführt und relativiert. Diese Kritik spielt auch in der Parlamentsdebatte eine Rolle: Grüne und Linke kritisieren die Regierung intensiv. Sie werfen ihr vor, zu langsam, teilweise sogar kontraproduktiv zu handeln. Doch trotz dieser Überschneidungen in der oppositionellen und in der medialen Darstellung widerspricht diese Kategorie der Indexing-Hypothese - die Medien gehen an entscheidenden Punkten deutlich über die politische Darstellung hinaus.

\subsection{Nachhaltigkeitsbegriff als tendenziell leerer Signifikant}

Insgesamt entsteht der Eindruck, dass der Nachhaltigkeitsbegriff innerhalb der politischen Eliten anerkannt ist. In der Medienberichterstattung ist das weniger eindeutig. Der dort thematisierte Gebrauch zu Imagezwecken deutet darauf hin, dass der Begriff von einigen Unternehmen ausgehöhlt wird. Auch deutsche Politiker*innen neigen der Berichterstattung zufolge dazu, Dinge als nachhaltig zu bezeichnen, die aus der Sicht anderer Akteur*innen nicht oder kaum nachhaltig sind.

Es gibt also eine große Bandbreite an Nachhaltigkeitsbehauptungen, die qualitativ erhebliche Unterschiede aufweisen können. Die Vielzahl 
an möglichen Definitionen wird in den analysierten Artikeln mehrfach kritisiert. Sie gilt als Hindernis für diejenigen, die eine nachhaltige Entwicklung tatsächlich vorantreiben wollen. Aufgrund der intransparenten und vagen Nutzung des Begriffs können Konsument*innen demnach oft nicht wissen, woran sie sich orientieren sollen und welchen Versprechen sie glauben können:

Es fängt damit an, dass er [der aufgeklärte Konsument; MC] nicht mehr weiß, welchen Informationen er noch vertrauen kann. Ist da, wo Bio draufsteht, auch wirklich Bio drin? Kann es stimmen, dass die Lebensmittel aus Italien geliefert wurden? Und wie sollen Bio und China bitte schön zusammengehen? Informieren kann man sich heute über die Herkunft der Produkte rund um die Uhr. Das Smartphone liegt immer in erreichbarer Nähe. Aber sind die Informationen nicht interessengeleitet und manipuliert? (Kläsgen 2017)

\section{Tab. 7: Zusammenfassung der Analysekategorie » Nachhaltigkeits-} begriff als tendenziell leerer Signifikant«.

\begin{tabular}{l|l|l|}
$\begin{array}{l}\text { Gemeinsame } \\
\text { Darstellungen }\end{array}$ & Keine. \\
\hline $\begin{array}{l}\text { Exklusive } \\
\text { Darstellungen }\end{array}$ & $\begin{array}{l}\text { Medien: Vieles, was als nachhaltig } \\
\text { bezeichnet wird, ist nicht wirklich } \\
\text { nachhaltig; Nachhaltigkeitsbegriff } \\
\text { sorgt derzeit für Verwirrung und } \\
\text { Vertrauensverlust unter Kundin- } \\
\text { nen und Anleger*innen; Nachhal- } \\
\text { tigkeitsbegriff ist möglicherweise } \\
\text { sinnlos. }\end{array}$ & \\
\hline & Kein Indexing. & \\
\hline
\end{tabular}

In der Berichterstattung wird der Begriff Nachhaltigkeit teilweise als tendenziell leerer Signifikant präsentiert, wenn auch nicht vollends in diesen Status erhoben. Ein großer Teil der medialen Darstellung deckt sich mit Giulios $(2004,11)$ Feststellung, dass der Nachhaltigkeitsbegriff zu einem »leeren Modewort « (ebd.) werden könnte. An dieser Stelle treten die Unterschiede zwischen der medialen und der politischen Darstellung klar hervor: Diese von den Medien thematisierte Problematik kommt in der Bundestagsdebatte so gut wie nicht vor. Es handelt sich um ein weiteres Beispiel dafür, dass die Medienberichterstattung an entscheidenden Stellen deutlich über die politische Darstellung hinausgeht. 


\section{$7 \quad$ Fazit}

Im Rahmen der untersuchten Nachhaltigkeitsdebatte gibt es große Überschneidungen zwischen den politischen und medialen Darstellungen. Sie zeigen, dass es durchaus eine gemeinsame Basis an Perspektiven gibt, die Politiker*innen und Qualitätsmedien nach außen kommunizieren. Der UN-Nachhaltigkeitsbegriff etwa ist weitgehend anerkannt, genauso wie die gemeinsame Verantwortung von Politik, Wirtschaft, Wissenschaft und Gesellschaft für die nachhaltige Entwicklung. Mehrere Darstellungen widersprechen aber der abgeschwächten Variante der Indexing-Hypothese, die davon ausgeht, dass die politischen und medialen Darstellungen größtenteils deckungsgleich sind. So beschäftigen sich die analysierten Medien mit dem Thema Greenwashing, das in der Bundestagsdebatte keine Rolle spielt, und gehen deutlich über die politische Darstellung hinaus, wenn sie über den Verzicht als Alternative zum nachhaltigen Konsum berichten und den Nachhaltigkeitsbegriff als inhaltsleer einordnen. Die Frage, ob Deutschland ein internationaler Vorreiter in der nachhaltigen Entwicklung ist, taucht wiederum nur in der Bundestagsdebatte, nicht aber in der untersuchten Berichterstattung auf.

Die Abweichungen der beiden Darstellungen voneinander sind im untersuchten Fall an entscheidenden Stellen zu groß, um von Indexing zu sprechen. Stattdessen untermauern die Ergebnisse Krügers (2016, 27) These, dass Politiker*innen und Journalist*innen teilweise abhängig, teilweise unabhängig voneinander agieren. Der qualitative Analyseansatz hat sich an dieser Stelle als hilfreich erwiesen. Er ermöglicht, komplexe Sinnzusammenhänge zu erkennen, die in einer quantitativen Analyse untergehen können. Allerdings hat sich auch gezeigt, dass die vielen Uneindeutigkeiten in der Nachhaltigkeitsdebatte die Komplexität der qualitativen Analyse drastisch erhöhen. Um Indexing noch genauer zu untersuchen, wäre es ratsam, qualitative und quantitative Ansätze stärker miteinander zu verknüpfen. Eine interessante Fragestellung bestünde darin, ob die Analyse aktueller Darstellungen die hier vorgestellten Ergebnisse stützt beziehungsweise wie sich die politischen und medialen Darstellungen seit Anfang 2017 verändert haben - sei es durch das Aufkommen von Fridays for Future, die Corona-Pandemie oder die Einzüge der AfD und der FDP in den Bundestag.

Für den Qualitätsjournalismus in Deutschland zeigt die Analyse, dass die untersuchten Medien im Falle der Nachhaltigkeitsdebatte größtenteils ihrer Aufgabe nachkommen, kritisch und offen zu berichten. Aller- 
dings wäre es Aufgabe der Presse, über die Vorreiterdebatte zu schreiben und sie einzuordnen. Hier zeigt sich, dass Indexing stellenweise wünschenswert sein könnte: Die Berichterstattung über die Debatte ist Voraussetzung für eine tiefergehende Auseinandersetzung mit relevanten Fragen: Ist Deutschland in der nachhaltigen Entwicklung tatsächlich internationaler Vorreiter? Und welche Rolle spielt dieser Umstand?

Bemerkenswert ist, dass die Medien sich intensiv dem Komplex »Verzicht versus nachhaltiger Konsum« widmen. Denn die Annahme, dass wir in der nachhaltigen Entwicklung notwendigerweise auf Dinge verzichten müssen, widerspricht dem Drei-Säulen-Konzept und befürwortet das, was die jungen Klimaaktivist*innen auf ihren Freitagsdemos fordern. Aus dieser Perspektive ist die gleichwertige Behandlung von Umwelt, Sozialem und Wirtschaft nicht mehr zeitgemäß.

\section{Literatur}

Adam, Silke. 2008. Massenmedien als Herausforderer oder Agenturen nationaler Eliten? Eine Analyse der deutschen und französischen EU-Erweiterungsdebatte. In: Massenmedien als politische Akteure. Konzepte und Analysen, herausgegeben von Barbara Pfetsch und Silke Adam, 116-143. Wiesbaden: VS Verlag für Sozialwissenschaften.

Bauchmüller, Michael, und Kristiana Ludwig. 2017. Verzichten können andere. Süddeutsche Zeitung vom 16. März, https://www.sueddeutsche.de/wirtschaft/ umweltpolitik-verzichten-koennen-andere-1.3422358. Zugegriffen: 28. Juni 2018.

Bennett, W. Lance. 1990. Toward a Theory of Press-State Relations in the United States. Journal of Communication 40 (2): 103-125.

Bennett, W. Lance. 2009. Power and the News Media: The Press and Democratic Accountability. In: Politik in der Mediendemokratie, herausgegeben von Frank Marcinkowski und Barbara Pfetsch, 84-102. Wiesbaden: VS Verlag für Sozialwissenschaften.

Bennett, W. Lance, und Steven Livingston. 2003. Editors' Introduction: A Semi-Independent Press: Government Control and Journalistic Autonomy in the Political Construction of News. Political Communication 20 (4): 359-362.

Bilharz, Michael. 2005. Nachhaltiger Konsum: Die Suche nach dem nächsten Schritt. Consumer Science 5. http://www.keypointer.de/fileadmin/media/Bilharz_2005_Nachhaltiger-Konsum-die-Suche-nach-dem-nächsten-Schritt_Bericht.pdf. Zugegriffen: 22. November 2020.

Bilharz, Michael. 2008. »Key Points« nachhaltigen Konsums. Ein strukturpolitisch fundierter Strategieansatz für die Nachhaltigkeitskommunikation im Kontext aktivierender Verbraucherpolitik. Marburg: Metropolis.

Brand, Karl-Werner, und Georg Jochum. 2000. Der deutsche Diskurs zu nachhalti- 
ger Entwicklung. Abschlussbericht eines DFG-Projekts zum Thema »Sustainable Development/Nachhaltige Entwicklung - Zur Sozialen Konstruktion Globaler Handlungskonzepte im Umweltdiskurs.«MPS-Texte 1.

Brand, Ulrich, und Christoph Görg. 2002. »Nachhaltige Globalisierung«? Sustainable Development als Kitt des neoliberalen Scherbenhaufens. In: Mythen globalen Umweltmanagements, herausgegeben von Christoph Görg und Ulrich Brand, 12-47. Münster: Westfälisches Dampfboot.

Brüggemann, Michael, und Hartmut Weßler. 2009. Medien im Krieg. Das Verhältnis von Medien und Politik im Zeitalter transnationaler Konfliktkommunikation. In: Politik in der Mediendemokratie, herausgegeben von Frank Marcinkowski und Barbara Pfetsch, 635-657. Wiesbaden: VS Verlag für Sozialwissenschaften.

Bundesregierung. 2017. Deutsche Nachhaltigkeitsstrategie. Neuauflage 2016. https://www.bundesregierung.de/Content/Infomaterial/BPA/Bestellservice/ Deutsche_Nachhaltigkeitsstrategie_Neuauflage_2016.pdf. Zugegriffen: 22. November 2020.

Bundesregierung. 2020. Neue Kraftstoffe und Antriebe. https://www.bundesregierung.de/breg-de/themen/neue-kraftstoffe-und-antriebe-994216. Zugegriffen: 22. November 2020.

Dahrendorf, Ralf. 1968. Festansprache: Professor Ralf Dahrendorf. „Demokratie glaubwürdig machen«. In: Theodor-Heuss-Preis 1968. Demokratie glaubwürdig machen, herausgegeben von Waldemar Besson, Adolf Butenandt, Ralf Dahrendorf, Hildegard Hamm-Brücher und Karin Storch, 13-21. https://theodorheuss-stiftung.de/wp-content/uploads/Jahresband-1968.pdf. Zugegriffen: 22. November 2020.

Deutscher Bundestag. 2017. Stenografischer Bericht. 229. Sitzung. Protokoll 18/229. http://dip21.bundestag.de/dip21/btp/18/18229.pdf. Zugegriffen: 1. August 2018.

Eckl, Christian. 2011. Wie unabhängig von politischen Eliten sind die Printmedien? https://refubium.fu-berlin.de/handle/fub188/6071. Zugegriffen: 22. November 2020.

Franke, Ulrich, und Ulrich Roos. 2010. Rekonstruktionslogische Forschungsansätze. In: Handbuch der Internationalen Politik, herausgegeben von Carlo Masala, Frank Sauer und Andreas Wilhelm, 285-303. Wiesbaden: VS Verlag.

Gerber, Claus-Peter. 1968. Soziale Strukturen und Interaktionsmuster. In: Die Massenmedien und die Organisation politischer Interessen, herausgegeben von ClausPeter Gerber und Manfred Stosberg, 61-105. Bielefeld: Bertelsmann Universitätsverlag.

Gerhards, Jürgen, Friedhelm Neidhardt, und Dieter Rucht. 1998. Zwischen Palaver und Diskurs. Strukturen öffentlicher Meinungsbildung am Beispiel der deutschen Diskussion zur Abtreibung. Opladen: Westdeutscher Verlag.

Giulio, Antonietta Di. 2004. Die Idee der Nachhaltigkeit im Verständnis der Vereinten Nationen. Anspruch, Bedeutung und Schwierigkeiten. Münster: Lit.

Heine, Matthias. 2017. »Nachhaltig« ist unser »Halal«. Die Welt vom 5. Juni, https:// www.welt.de/debatte/kommentare/article165225725/Nachhaltig-ist-unserhalal.html. Zugegriffen: 22. November 2020. 
Herman, Edward, und Noam Chomsky. 1988. Manufacturing Consent. The Political Economy of the Mass Media. New York: Pantheon.

Herzog, Dietrich, und Bernhard Weßels. 2005. Lexikon der Politikwissenschaft: Theorien, Methoden, Begriffe. In: Lexikon der Politikwissenschaft: Theorien, Methoden, Begriffe. Band 2: N-Z, herausgegeben von Dieter Nohlen und RainerOlaf Schultze, 732-736. München: C. H. Beck.

Hille, Steven. 2017. Was bringen Ökolabels im Tourismus? Die Welt vom 9. Juni, https://www.welt.de/reise/article165296995/Was-bringen-Oekolabels-imTourismus.html. Zugegriffen: 28. Juni 2018.

Infratest Dimap. 2020. Glaubwürdigkeit der Medien 2020. https://www.infratestdimap.de/umfragen-analysen/bundesweit/umfragen/aktuell/glaubwuerdigkeit-der-medien-2020/. Zugegriffen: 22. November 2020.

Jandura, Olaf, und Raphael Kösters. 2017. Neue Medienumgebungen, andere Auswahlkriterien? Überlegungen zur Auswahl von Medienangeboten bei Inhaltsanalysen. Publizistik 62 (1): 25-41.

Kabalak, Alihan, Birger Priddat, und Markus Rohmberg. 2008. Medien als Schnittstelle zwischen politischen und ökonomischen Strukturen - Politische Kommunikation in der Perspektive der Institutionenökonomie. In: Massenmedien als politische Akteure. Konzepte und Analysen, herausgegeben von Barbara Pfetsch und Silke Adam, 52-72. Wiesbaden: VS Verlag für Sozialwissenschaften.

Kläsgen, Michael. 2017. Die Leiden des aufgeklärten Konsumenten. Süddeutsche Zeitung vom 1. Januar, https://www.sueddeutsche.de/wirtschaft/nachhaltigeinkaufen-die-leiden-des-aufgeklaerten-konsumenten-1.3315584. Zugegriffen: 28. Juni 2018.

Kresta, Edith. 2017. Party ohne Ende. taz vom 19. März, https://www.taz.de/Archiv-Suche/!5390075. Zugegriffen: 28. Juni 2018.

Krüger, Uwe. 2019. Meinungsmacht. Der Einfluss von Eliten auf Leitmedien und Alpha-Journalisten - Eine kritische Netzwerkanalyse. 2., überarbeitete und erweiterte Auflage. Köln: Herbert von Halem.

Krüger, Uwe. 2016. Medien im Mainstream - Problem oder Notwendigkeit? Aus Politik und Zeitgeschichte 66 (30-32): 22-27.

Lang, Kurt, und Gladys Engel Lang. 2004. Noam Chomsky and the Manufacture of Consent for American Foreign Policy. Political Communication 21 (1): 93-101.

Lüter, Albrecht. 2008. Die Kommentarlage. Profilbildung und Polyphonie in medienöffentlichen Diskursen. Wiesbaden: VS Verlag für Sozialwissenschaften.

Maurer, Torsten, Jens Vogelgesang, Moritz Weiß, und Hans-Jürgen Weiß. 2008. Aktive oder passive Berichterstatter? Die Rolle der Massenmedien während des Kosovo-, Afghanistan- und Irakkriegs. In: Massenmedien als politische Akteure. Konzepte und Analysen, herausgegeben von Barbara Pfetsch und Silke Adam, 144-170. Wiesbaden: VS Verlag für Sozialwissenschaften.

Mautz, Rüdiger, Andreas Byzio, und Wolf Rosenbaum. 2008. Auf dem Weg zur Energiewende. Die Entwicklung der Stromproduktion aus erneuerbaren Energien in Deutschland. Göttingen: Universitätsverlag Göttingen.

Mothes, Cornelia. 2014. Objektivität als professionelles Abgrenzungskriterium im 
Journalismus. Eine dissonanztheoretische Studie zum Informationsverhalten von Journalisten und Nicht-Journalisten. Baden-Baden: Nomos.

Neuberger, Christoph, und Peter Kapern. 2013. Grundlagen des Journalismus. Wiesbaden: Springer VS.

Paqué, Karl-Heinz. 2012. Wert des Wachstums: Kompass für eine Kontroverse. Aus Politik und Zeitgeschichte 27-28: 15-19.

Parisi, Peter. 1997. Toward a »Philosophy of Framing«: News Narratives for Public Journalism. Journalism \& Mass Communication Quarterly 74 (4): 673-686.

Pfetsch, Barbara, und Frank Marcinkowski. 2009. Problemlagen der »Mediendemokratie«. Theorien und Befunde zur Medialisierung von Politik. In: Politik in der Mediendemokratie, herausgegeben von Frank Marcinkowski und Barbara Pfetsch, 11-33. Wiesbaden: VS Verlag für Sozialwissenschaften.

Rickert, Heinrich. 1929. Zur Lehre von der Definition. 3., verbesserte Auflage. Tübingen: J. C.B. Mohr.

RNE - Rat für Nachhaltige Entwicklung. 2018. Auftrag an den Rat für nachhaltige Entwicklung. https://www.nachhaltigkeitsrat.de/der-rat/auftrag-des-rates/. Zugegriffen: 25. Juli 2018.

RNE - Rat für Nachhaltige Entwicklung. 2020. Nachhaltige Entwicklung. https:// www.nachhaltigkeitsrat.de/nachhaltige-entwicklung/. Zugegriffen: 22. November 2020.

Samanta, Sayan. 2016. Priority Setting - or Rather Not? Positive Concerns for SDGs. http://www.die-gdi.de/uploads/media/German_Development_Institute_Samanta_12.09.2016.pdf. Zugegriffen: 22. November 2020.

Santarius, Tilman. 2012. Der Rebound-Effekt. Über die unerwünschten Folgen der erwünschten Energieeffizienz. Wuppertal: Wuppertal Institut für Klima, Umwelt, Energie GmbH.

Schafhausen, Franzjosef. 2013. Die Energiewende - Aufbruch in Die Zukunft. Vierteljahreshefte zur Wirtschaftsforschung 82 (3): 11-28.

Seefried, Elke. 2015. Rethinking Progress. On the Origin of the Modern Sustainability Discourse, 1970-2000. Journal of Modern European History 13 (3): 377399.

Soentgen, Jens. 2016. Nachhaltigkeit als Nießbrauch. Das römische Rechtsinstitut des usus fructus und seine systematische Bedeutung für das Konzept der nachhaltigen Nutzung. GAIA - Ecological Perspectives for Science and Society 25 (2): 117-125.

Steiner, Adrian, und Otfried Jarren. 2009. Intermediäre Organisationen unter Medieneinfluss? Zum Wandel der politischen Kommunikation von Parteien, Verbänden und Bewegungen. In: Politik in der Mediendemokratie, herausgegeben von Frank Marcinkowski und Barbara Pfetsch, 251-269. Wiesbaden: VS Verlag für Sozialwissenschaften.

Strübing, Jörg. 2008. Grounded Theory. Zur sozialtheoretischen und epistemologischen Fundierung des Verfahrens der empirisch begründeten Theoriebildung. Wiesbaden: VS Verlag.

UN-Generalversammlung. 2015. Transforming Our World: The 2030 Agenda for 
Sustainable Development. http://www.un.org/ga/search/view_doc.asp?symbol=A/RES/70/1\&Lang=E. Zugegriffen: 22. November 2020.

Voltmer, Katrin. 2008. »Vierte Gewalt« im Schatten der Vergangenheit - Die Transformation der Massenmedien in Neuen Demokratien. In: Massenmedien als politische Akteure. Konzepte und Analysen, herausgegeben von Barbara Pfetsch und Silke Adam, 92-115. Wiesbaden: VS Verlag für Sozialwissenschaften.

WCED. 1987. Report of the World Commission on Environment and Development: Our Common Future. http://www.un-documents.net/our-common-future.pdf. Zugegriffen: 22. November 2020.

Weischenberg, Siegfried, Armin Scholl, und Maja Malik. 2006. Die Souffleure der Mediengesellschaft: Report über die Journalisten in Deutschland. Konstanz: UVK.

Welfens, Paul J.J. 2011. Überwindung der Banken- und Finanzkrise: Optionen der Wachstums- bzw. Wirtschaftspolitik. In: Zukunftsfähige Wirtschaftspolitik für Deutschland und Europa, herausgegeben von Paul J.J. Welfens, 1-74. Heidelberg: Springer.

Wille, Joachim. 2017. Wem gehört der letzte Tropfen? Frankfurter Rundschau vom 13. Juni, https://www.fr.de/wirtschaft/gehoert-letzte-tropfen-11081921.html. Zugegriffen: 28. Juni 2018.

Willmroth, Jan. 2017. Mehr als grünes Geschwätz. Süddeutsche Zeitung vom 25. Juni, https://www.sueddeutsche.de/wirtschaft/geldanlage-mehr-als-gruenes-geschwaetz-1.3559816. Zugegriffen: 28. Juni 2018.

Zürcher, Ulrich. 1965. Die Idee der Nachhaltigkeit unter spezieller Berücksichtigung der Gesichtspunkte der Forsteinrichtung. ETH Zürich. https://www.research-collection.ethz.ch/handle/20.500.11850/132556. Zugegriffen: 22. November 2020.

\section{Open Access}

Dieser Beitrag erscheint unter der Creative-Commons-Lizenz CC BY-ND 3.0 DE: https://creativecommons.org/licenses/by-nd/3.0/de/. 